\title{
Patterns of telomere length with age in African mole-rats: New insights from quantitative fluorescence in situ hybridisation (qFISH)
}

\author{
Stephanie R.L. Leonida ${ }^{1,2}$, Nigel C. Bennett ${ }^{2}$, Andrew R. Leitch ${ }^{1}$, Chris G. Faulkes ${ }^{\text {Corresp. } 1}$ \\ ${ }^{1}$ School of Biological \& Chemical Sciences, Queen Mary University of London, London, United Kingdom \\ 2 Department of Zoology, University of Pretoria, Pretoria, South Africa \\ Corresponding Author: Chris G. Faulkes \\ Email address: c.g.faulkes@qmul.ac.uk
}

Naked mole-rats Heterocephalus glaber (NMRs) are the longest-lived rodent and also resist the normal signs of senescence. In a number of species, cellular ageing has been correlated with a reduction in telomere length, yet relatively little is known about telomeres and their age-related dynamics in NMRs and other African mole-rats. Here, we apply fluorescence in situ hybridisation (FISH) to quantify telomeric repeat sequences in the NMR, the Damaraland mole-rat, Fukomys damarensis (DMR) and the Mahali mole-rat, Cryptomys hottentotus mahali (MMR). Both terminal and non-terminal telomeric sequences were identified in chromosomes of the NMR and DMR, whilst the MMR displayed only terminal telomeric repeats. Measurements of tooth wear and eruption patterns in wild caught DMRs and MMRs, and known ages in captive bred NMRs, were used to place individuals into relative age classes and compared with a quantitative measure of telomeric fluorescence (as a proxy for telomere size). While NMRs and MMRs failed to show an age-related decline in telomeric fluorescence, the DMR had a significant decrease in fluorescence with age, suggesting a decrease in telomere size in older animals. Our results suggest that among African mole-rats there is variation between species with respect to the role of telomere shortening in ageing, and the replicative theory of cellular senescence. 
1 Patterns of telomere length with age in African mole-rats: new insights from quantitative

2 Fluorescence In Situ Hybridisation (qFISH)

3

4 Stephanie R.L. Leonida ${ }^{1,2}$, Nigel C. Bennett ${ }^{2}$ Andrew R. Leitch ${ }^{1}$ \& Chris G. Faulkes $^{1}$

5

$6{ }^{1}$ Queen Mary University of London, School of Biological and Chemical Sciences, UK

$7{ }^{2}$ Mammal Research Institute, Department of Zoology and Entomology, University of Pretoria,

8 Pretoria 0002, South Africa

9

10

11

12

13

14

15

16 


\section{Abstract}

18 Naked mole-rats Heterocephalus glaber (NMRs) are the longest-lived rodent and also resist the normal signs of senescence. In a number of species, cellular ageing has been correlated with a reduction in telomere length, yet relatively little is known about telomeres and their age-related dynamics in NMRs and other African mole-rats. Here, we apply fluorescence in situ hybridisation (FISH) to quantify telomeric repeat sequences in the NMR, the Damaraland molerat, Fukomys damarensis (DMR) and the Mahali mole-rat, Cryptomys hottentotus mahali (MMR). Both terminal and non-terminal telomeric sequences were identified in chromosomes of the NMR and DMR, whilst the MMR displayed only terminal telomeric repeats. Measurements of tooth wear and eruption patterns in wild caught DMRs and MMRs, and known ages in captive bred NMRs, were used to place individuals into relative age classes and compared with a quantitative measure of telomeric fluorescence (as a proxy for telomere size). While NMRs and MMRs failed to show an age-related decline in telomeric fluorescence, the DMR had a significant decrease in fluorescence with age, suggesting a decrease in telomere size in older animals. Our results suggest that among African mole-rats there is variation between species with respect to the role of telomere shortening in ageing, and the replicative theory of cellular senescence.

\section{Introduction}

In most eukaryotes, telomere length reduction with age is a direct outcome of incomplete replication of chromosome ends during replication; the 'end replication problem' (Lansdorp et al., 1996; Zakian et al., 2012). Once telomeres reach a critically short length, cellular signals initiate the process of cell cycle arrest and subsequent senescence, known as the "replicative theory of 
40 ageing/senescence". Studies of telomere length dynamics in humans and birds have supported this

41 theory, finding a strong correlation between telomere length and lifespan, with a decrease in

42 telomere length with age (Vleck, 2003; Harley et al., 1990; Haussmann et al., 2003; Heidinger et

43 al., 2012). Telomere-dependent replicative senescence is also seen as an anti-cancer mechanism

44 in humans, as progressive telomere shortening ultimately leads to permanent arrest of cell

45 proliferation. While in some species there is a good evidence for a link between telomere length

46 and life history, it is less clear whether there is a direct causal role between them.

It is thought that long-lived species have evolved a suppression of telomerase activity to mediate replicative senescence. It is argued that there has been selection in larger animals (which tend to live longer) to have evolved suppressed telomerase activity to alleviate an increased cancer risk arising from their large number of cells and increased lifespan, which provides increased opportunities for cancer-inducing mutations to occur ("Peto's paradox"). This hypothesis has been suggested to apply generally in mammals (Gomes et al., 2011), including rodent species (Seluanov et al., 2007), where telomerase activity has been shown to correlate with body mass.

Naked mole-rats (NMRs) may be an anomaly to Peto's hypothesis- they are a small rodent with extreme longevity (potentially living up to 33 years), but with high levels of telomerase activity (Tian et al., 2018). Alternative telomere-independent mechanisms appear to have evolved in NMR to suppress cancer-inducing mutations (Gorbunova et al., 2014; Tian et al., 2017) and to resist the normal signs of senescence (Buffenstein, 2005; Buffenstein, 2008) over their lifespan. This paper examines NMRs and two other species of African mole-rats in the family Bathyergidae with different longevities to compare age-related dynamics in telomere length: Fukomys damarensis (or Damaraland mole-rat, DMR) and Cryptomys hottentotus mahali (or Mahali mole- 
63 technique of quantitative peptide nucleic acid-FISH (PNA-QFISH) to (i) identify terminal and/or

64 non-terminal telomeric repeats in the NMR, DMR and MMR, and (ii) quantify changes in telomere

65 size with animal age.

67 Materials and Methods

68

69

\section{Animals}

Animals were caught using Hickman traps and baited with sweet potato (Hickman, 1979; Arslan, 2013). A total of 12 DMRs were used in this study (4 Males and 8 females), captured on a farm near Tswalu Kalahari Reserve, South Africa $\left(27.2961^{\circ} \mathrm{S} 22.3943^{\circ} \mathrm{E}\right)$, with permission from Department of Environment and Nature Conservation, Northern Cape Province (Permit No: FAUNA 171/2/2015). A total of 27 MMRs (13 Males and 14 Females) were caught on private plots in northern Pretoria $\left(25.6567^{\circ} \mathrm{S} 27.9547^{\circ} \mathrm{E}\right)$, with permission from Gauteng Department of Nature Conservation (Permit number: CPF6-0127). The study was approved through the Animal Ethics Committee, University of Pretoria, South Africa, AUCC project number: EC037-15. For NMRs, samples from 13 animals (8 Males and 5 Females) were obtained from captive bred colonies kept at Queen Mary University of London. Tissue was obtained from post-mortem specimens from animals free from disease in compliance with national (Home Office) and institutional procedures and guidelines.

S1 Stage of molar tooth wear and eruption pattern was used to place individual DMRs and MMRs into one of five, or one of four age classes in order to give an approximation of age as described previously for DMRs and Cryptomys hottentotus (Bennett et al., 1990). NMRs of known ages (from birth records) together with the DMRs and MMRs of estimated ages were placed into 
85

86

87

an age category of young, middle-aged or old to enable a more standardised comparison for further analysis (Table S1). No samples were obtained from middle-aged DMRs.

\section{Bone marrow extraction and FISH}

Bone marrow lymphocyte cells were extracted using a standard protocol (https://www.jax.org/research-and-faculty/tools/cytogenetic-and-down-syndrome-modelsresource/protocols/marrow-preps-protocol) from all 12 DMRs, all 27 MMRs and 9 of the 13 NMRs. Because of their smaller size, four of the NMR samples failed to yield sufficient cells for further analysis. Temperature for incubation of bone marrow cells was modified to mimic the internal body temperature of each mole-rat species; $32{ }^{\circ} \mathrm{C}(\mathrm{NMR})$ and $35{ }^{\circ} \mathrm{C}(\mathrm{MMR}$ and DMR). Cells were prepared and stained with DAPI and Cy3 PNA probe with Telomere PNA FISH Kit (K5326; DAKO, Glostrup, Denmark) using the protocol provided. A mixed population of lymphocyte cells (50 cell replicates for each animal sampled), each being diploid un-replicated cells (2C value) were chosen from each sample preparation for fluorescence analysis.

\section{Microscope and image capture}

FISH was conducted on interphase cells and representative metaphase spreads and quantitative FISH (qFISH) measurements of total telomere fluorescence of interphase cells from DMR, MMR and NMR were acquired on a Leica DMRA2 upright microscope equipped with a CoolSNAP HQ CCD camera using the 100x 1.4 NA Oil immersion objective, with DAPI and Cy3 filters. The aperture window was set at $1.25 \mathrm{x}$ and kept constant during image capture. The exposure time was kept constant for each species (330 ms for DMR and NMR; $470 \mathrm{~ms}$ for MMR), when using the 
106 Cy3 filter for image capture of telomere spots of interphase cells and metaphase spreads. Telomere

107 fluorescence was measured using OpenLab version 5.5.0.

The mean telomere fluorescence was measured for 50 randomly selected nonoverlapping, circular or near circular interphase cells from each individual for each species. Samples from each species were batch processed together to mitigate against inter-assay variation. To verify that cells were in a state where chromosomal material remained unreplicated, measurements of mean DAPI fluorescence were recorded (cells with double the amount of fluorescence would have replicated their chromosomal material). DAPI fluorescence measurements confirmed that cells chosen for telomere fluorescence measurements had not replicated their DNA, i.e. they were in G1 phase of interphase.

\section{Calculation of total telomere fluorescence}

117 A background measurement of a blank area (not containing interphase cells and that of a specified area) was taken for each image to be analysed. The area of this measurement was kept constant for each image captured for consistency across samples. The mean background measurement was then subtracted from the mean fluorescence of telomere spots to produce the total telomere fluorescence "volume" (intensity x area) expressed in arbitrary units for a given cell.

\section{Statistical analysis}

123 All analyses were conducted using the R statistical software programme (Version 3.2.5). None of

124 the fluorescence data (for the NMR, DMR and MMR) satisfied the criteria of a normal distribution required for a two-level nested ANOVA. Despite the data being transformed by a number of

126 methods, it remained positively skewed and divergent from normality (Shapiro-Wilk test: $\mathrm{p}<$

127 0.0001). To overcome this and increase robustness, the data were ranked to decrease the within 
128 group variance, and total lymphocyte telomere signal fluorescence was tested against age class 129 using a nested ANOVA. Within the nested ANOVA model, the total volume of telomere

130 fluorescence was input as the response variable, with the relative age class and individual identity 131 input as explanatory variables. A Tukey post-hoc test was used to assess the difference in variation 132 between telomere fluorescence of young, middle-aged and old MMRs

\section{Results}

Terminal telomeric repeats were successfully detected and visualised in the DMR, MMR and NMR (Figure 1). Two homologous chromosome pairs in the DMR clearly share ITSs along their chromosome arms, illustrated by sections of diffuse qFISH staining. Additionally, a single homologous chromosome pair appears to have a shorter tract of these repeat sequences (fainter staining area seen in some cells; Figure 1B). The NMR potentially had ITSs on one chromosome pair (Figure 1D), although the region of increased fluorescence was faint, but noticable. In contrast, MMR chromosomes appeared to display a strictly terminal telomeric morphology, with no apparent ITSs observed on any chromosomes (Figure 1C). with previous studies (Nevo et al., 1986). We confirmed that the MMR had the same chromosome number as other mole-rats in the Cryptomys hottentotus clade $(2 \mathrm{n}=54$ for females, and 53 for males; Figure S1) including Cryptomys h. hottentotus, Cryptomys h. natalensis and Cryptomys $h$.

147 pretoriae (Nevo et al., 1986; Faulkes et al., 2004; Deuve et al., 2008).

In DMRs, although there was a significant difference in total fluorescence among 
150 reduction in total telomere fluorescence in the bone marrow cells of old DMRs compared with

151 young animals $\left(\mathrm{F}_{2,1371}=10.14, \mathrm{p}<0.001\right.$; Figure 2A). For the MMR, significant variation in

152 telomere fluorescence of bone marrow cells was found between individual MMRs within each

153 group $\left(\mathrm{F}_{24,1371}=27.05, \mathrm{p}<0.001\right)$, and among groups of young, middle-aged and old individuals

$154\left(\mathrm{~F}_{2,1371}=10.14, \mathrm{p}<0.001 ;\right.$ Figure $\left.2 \mathrm{~b}\right)$. A post-hoc Tukey test a small, but significant increase in

155 telomere fluorescence in old versus middle-aged and young MMRs ( $\mathrm{p}<0.001$ and $\mathrm{P}<0,05$

156 respectively; Figure 2B). Finally, as with the other two species, NMRs had significant differences

157 in telomere fluorescence within age classes $\left(F_{6,441}=12.01, p<0.001\right)$, and between classes $\left(F_{2,441}\right.$

$158=23.90, \mathrm{p}<0.001)$. Total telomere fluorescence did not decline with age - old versus young

159 animals were not significantly different $(\mathrm{p}=0.21)$, while middle aged animals had a significant

160 increase in fluorescence compared to young and old age classes $(\mathrm{p}<0.001)$. Individual animal

161 sample means with standard errors plotted against age in years are shown on Figure S2)

\section{Discussion}

The use of qFISH for calculating telomere length is well established as a research tool (e.g. in mouse strains: Zijlmans et al., 1997), comparable with telomere length measurement method using Southern blotting/in situ hybridization techniques and in some applications may be more precise than qPCR methods (Hultdin et al., 1998; Gutierrez-Rodrigues et al., 2014). Apart from mammals, qFISH has also been employed as a single technique estimator of telomere length in diverse vertebrate taxa such as fish (Panasiak et al., 2020) and reptiles (Olsson et al., 2020). PNA-qFISH clearly identified terminal telomeric repeats in the DMR, MMR and NMR and we report for the

171 first time interstitial telomeric repeat sequences (ITSs) in the DMR and tentatively in the NMR 172 (Figure 1A and 1D). Chromosomes containing ITSs as observed in the DMR and potentially the 
173 NMR may indicate active karyotype evolution, and potentially mark regions of fusion of ancestral

174 chromosomes or sites of double-strand break repair in unstable areas of the genome (Meyne et al.,

175 1990; Ruiz-Herrera et al., 2008). Such molecular instability can lead to further breakage or fission

176 and chromosome fusions. It is well known that the DMR karyotype is among one of the most

177 rearranged karyotypes amongst members of the family Bathyergidae (Deuve et al., 2008).

178 Variation in chromosome number to produce chromosomal races within the DMR have recorded $1792 \mathrm{n}$ varying from 74 to 78 (Nevo et al., 1986) and also $2 \mathrm{n}=80$ individuals can occur in different 180 geographic areas (Deuve et al., 2008). This chromosomal variation, coupled with the observation 181 of ITSs, suggest that the DMR genome has ongoing, active karyotype evolution, in contrast to the 182 fixed karyotypes seen in the MMR and others in the Cryptomys hottentotus clade (Deuve et al., 183 2008). Future work could involve using $\mathrm{G}$ and $\mathrm{C}$-banding techniques and chromosome painting 184 using whole chromosome paints to observe association between heterochromatic regions and ITS 185 and terminal telomeric repeats (Yang et al, 2004). This may allow more detailed insights into the 186 various types of past chromosomal rearrangements that have occurred in the DMR and other 187 species in the genus Fukomys, where there is extensive karyotypic variation among species (Van 188 Daele et al., 2004). 1C) is a feature that has been reported previously in other mammals and may arise through telomere - nuclear envelope interactions. Such interactions are proposed to protect against recombination of telomeric repeats during mitosis and are considered essential for correct meiotic pairing of homologous chromosomes during meiosis (Schober et al., 2009; Chikashige et al., 2006; Ding et al., 2007). 

reported in other species, and some studies have shown associations between early-life telomere length and individual life history e.g. European badgers Meles meles (Van Lieshout et al., 2019), and meerkats (Cram et al., 2017). The significance of individual variation in telomere sizes in mole-rats identified in our study cannot be addressed further without additional longitudinal studies using PCR-based techniques to quantify telomere size. We also found interesting species differences in the variation in telomere fluorescence with age, although a limitation of the study was being able to accurately age the wild caught DMRs and MMRs. Our results revealed a significant reduction in telomere fluorescence and by implication a reduction in telomere size with age class when comparing young and old DMRs (Figure 2A), suggesting that telomere shortening is a cause or biomarker for cellular senescence in this species. In contrast, both MMRs and NMRs did not show an age-related decline in total telomere fluorescence (Figure 2B and 2C). Moreover, in both species we observed an increase in telomere fluorescence with age compared to the young age category, although our sample size was small for the NMR. These observations do not support the hypothesis of cellular senescence in these species and support other indirect evidence for telomere maintenance with age published for the NMR. Seluanov et al. (2007) found that telomerase activity in NMRs was high, and comparable with other small (but short-lived) rodents

213 like mice and gerbils that have a high incidence of cancer. Furthermore Kim et al. (2011) showed 214 stable levels of TERT expression (that functions to maintain telomeres) regardless of age 215 (comparing a new-born, 4 and 20 yr old NMRs). Recently, Adwan Shekhidem et al. (2019) used a different (qPCR-based) approach to show that telomere length in the NMR did not shorten with age, instead showing a mild elongation. The telomeric fluorescence signal in the NMR here 
218 suggest elevated activity of telomere elongating processes in middle aged animals. Conversely, 219 Adwan Shekhidem et al. (2019) found that telomere length in the cancer resistant, long-lived blind 220 mole-rat Spalax declined with age, in a similar fashion to short-lived rodents, similar to our

221 observations here for DMR. Collectively, these results highlight some fascinating variation in 222 telomere dynamics within the African mole-rats, and among other rodents, revealing the potential 223 for evolutionary gains and losses of mechanisms associated with telomere maintenance, cancer 224 resistance, longevity and senescence.

\section{Acknowledgements}

227

Daniel Hart, Ruth Rose and Elizabeth Archer are thanked for their support and guidance in the lab. We thank Steve Le Comber, Sally Faulkner and Joanne Littlefair for advice and help with the statistical analysis.

\section{References}

Adwan Shekhidem H, Sharvit L, Leman E, Manov I, Roichman A, Holtze S, Atzmon G. 2019. Telomeres and longevity: A cause or an effect? International Journal of Molecular Sciences 20:E3233 DOI: 10.3390/ijms20133233.

Arslan A. 2013. A new live trap to catch blind mole-rats (Spalax sp.). Folia Zoologica 2:130-132

Azzalin CM, Nergadze SG, Giulotto E. 2001. Human intrachromosomal telomeric-like repeats: sequence organization and mechanisms of origin. Chromosoma 110:75-82 DOI: $10.1007 / \mathrm{s} 004120100135$. 
239 Baird DM, Rowson J, Wynford-Thomas D, Kipling D. 2003. Extensive allelic variation and

240 ultrashort telomeres in senescent human cells. Nature Genetics 33:203-207 DOI: 10.1038/ng1084.

241 Bennett NC, Jarvis JUM, Wallace DB. 1990. The relative age structure and body masses of 242 complete wild-captured colonies of two social mole-rats, the common mole-rat, Cryptomys 243 hottentotus hottentotus and the Damaraland mole-rat, Cryptomys damarensis. Journal of Zoology 244 220:469-485 DOI:10.1111/j.1469-7998.1990.tb04319.x.

245 Buffenstein R. 2005. The naked mole-rat: a new long-living model for human aging research. The 246 Journals of Gerontology. Series A, Biological Sciences and Medical Sciences 60:1369-1377 DOI: 247 10.1093/gerona/60.11.1369.

248

249

250

251

252

253

254

255

256

257

258

259

Buffenstein R. 2008. Negligible senescence in the longest living rodent, the naked mole-rat: insights from a successfully ageing species. Journal of Comparative Physiology (B) 178:439-455 DOI: $10.1007 / \mathrm{s} 00360-007-0237-5$.

Chikashige Y, Tsutsumi C, Yamane M, Okamasa K. 2006. Meiotic proteins bqt1 and bqt2 tether telomeres to form the bouquet arrangement of chromosomes. Cell 1:59-69 DOI: 10.1016/j.cell.2006.01.048.

Cram DL, Monaghan P, Gillespie R, Clutton-Brock T. 2017. Effects of early-life competition and maternal nutrition on telomere lengths in wild meerkats. Proceedings of the Royal Society B: Biological Sciences, 284:20171383 DOI: 10.1098/rspb.2017.1383.

Deuve LJ, Bennett NC, Britton-Davidian J, Robinson TJ. 2008. Chromosomal phylogeny and evolution of the African mole-rats (Bathyergidae) Chromosome Research 16:57-74 DOI: 10.1007/s10577-007-1200-8. 
260 Ding X, Xu R, Yu J, Xu T, Zhuang Y, Han M. 2007. SUN1 is required for telomere attachment

261 to nuclear envelope and gametogenesis in mice. Developmental Cell 12:863-872 DOI:

262 10.1016/j.devcel.2007.03.018.

263 Faulkes CG, Verheyen E, Verheyen W, Jarvis JU, Bennett NC. 2004. Phylogeographical

264 patterns of genetic divergence and speciation in African mole-rats (Family: Bathyergidae).

265 Molecular Ecology 3:613-629 DOI: 10.1046/j.1365-294X.2004.02099.x.

266 Gorbunova, V., Seluanov, A., Zhang, Z., Gladyshev, V. N., \& Vijg, J. 2014. Comparative 267 genetics of longevity and cancer: insights from long-lived rodents. Nature Reviews Genetics 15:126810 DOI: $10.1038 / \mathrm{nrg} 3728$.

269

270

271

272

273

274

275

276

277

278

279

280

281

Gomes NM, Ryder OA, Houck ML, Charter SJ, Walker W, Forsyth NR, Austad SN, Venditti

C, Pagel M, Shay JW, Wright WE. 2011. Comparative biology of mammalian telomeres: hypotheses on ancestral states and the roles of telomeres in longevity determination. Aging Cell 10:761-768 DOI:10.1111/j.1474-9726.2011.00718.x.

Gutierrez-Rodrigues F, Santana-Lemos BA, Scheucher PS, Alves-Paiva RM, Calado RT. 2014. Direct comparison of flow-FISH and $\mathrm{qPCR}$ as diagnostic tests for telomere length measurement in humans. PLoS One 9:e113747 DOI: 10.1371/journal.pone.0113747.

Haussmann MF, Vleck CM. 2002. Telomere length provides a new technique for aging animals. Oecologia 130:325-328 DOI: 10.1007/s00442-001-0827-y.

Haussmann MF, Vleck CM, Nisbet IC 2003 Calibrating the telomere clock in common terns, Sterna hirundo. Experimental Gerontology 38:787-789 DOI: 10.1016/S0531-5565(03)00109-8.

Harley CB, Futcher AB, Greider CW. 1990. Telomeres shorten during ageing of human fibroblasts. Nature 345:458-460 DOI: 10.1038/345458a0. 
282 Harley CB. 1995. Telomeres and Aging. In: Blackburn EH, Greider CW Eds. Telomeres. NY: 283 Cold Spring Harbor Laboratory Press, 247-263.

284

285

286

287

288

289

290

291

292

293

294

295

296

297

298

299

300

301

302

Hayflick L, Moorhead PS. 1961. The serial cultivation of human diploid cell strains. Experimental Cell Research 25:585-621 DOI: 10.1016/0014-4827(61)90192-6.

Heidinger BJ, Blount JD, Boner W, Griffiths K, Metcalfe NB, Monaghan P. 2012. Telomere length in early life predicts lifespan. Proceedings of the National Academy of Sciences of the United States of America 109:1743-1748 DOI: 10.1073/pnas.1113306109.

Hickman GC. 1979. A live-trap and trapping technique for fossorial mammals. South African Journal of Zoology 14:9-12.

Hultdin M, Grönlund E, Norrback K-F, Eriksson-Lindström E, Roos G, Just T. 1998. Telomere analysis by fluorescence in situ hybridization and flow cytometry, Nucleic Acids Research 26: 3651-3656 DOI: 10.1093/nar/26.16.3651.

Kim EB, Fang X, Fushan AA, Huang Z, Lobanov AV, Han L, Marino SM, Sun X, Turanov AA, Yang P, Yim SH, Zhao X, Kasaikina MV, Stoletzki N, Peng C, Polak P, Xiong Z, Kiezun A, Zhu Y, Chen Y, Kryukov GV, Zhang Q, Peshkin L, Yang L, Bronson RT, Buffenstein R, Wang B, Han C, Li Q, Chen L, Zhao W, Sunyaev SR, Park TJ, Zhang G, Wang J, Gladyshev VN. 2011. Genome sequencing reveals insights into physiology and longevity of the naked mole rat. Nature 479:223-7 DOI:10.1038/nature10533.

Lansdorp PM, Verwoerd NP, van de Rijke FM, Dragowska, V, Little M-T, Dirks RW, Raap AK Tanke HJ. 1996. Heterogeneity in telomere length of human chromosomes. Human Molecular Genetics 5:685-691 DOI: 10.1093/hmg/5.5.685. 
303

304

305

306

307

308

309

310

311

312

313

314

315

316

317

318

319

320

321

322

323

Meyne J, Baker R, Hobart HH, Hsu TC, Ryder OA, Ward OG, Wiley JE, Wurster-Hill DH, Yates TL, Moyzis RK. 1990. Distribution of non-telomeric sites of the (TTAGGG)n telomeric sequence in vertebrate chromosomes. Chromosoma 99:3-10 DOI: 10.1007/BF01737283.

Nergadze SG, Santagostino MA, Salzano A, Mondello C, Giulotto E. 2007. Contribution of telomerase RNA retrotranscription to DNA double-strand break repair during mammalian genome evolution. Genome Biology 12:R260 DOI: 10.1186/gb-2007-8-12-r260.

Nevo E, Capanna E, Corti M, Jarvis JUM, Hickman GC. 1986. Karyotype differentiation in the endemic subterranean Mole-rats of South Africa (Rodentia, Bathyergidae). Zeitschrift für Säugetierkunde 51:36-49

Olsson M, Geraghty NJ, Wapstra E, Wilson M. 2020. Telomere length varies substantially between blood cell types in a reptile. Royal Society Open Science 7:192136 DOI: 10.1098/rsos.192136.

Panasiak L, Dobosz S, Ocalewicz K. 2020. Telomere dynamics in the diploid and triploid rainbow trout (Oncorhynchus mykiss) assessed by Q-FISH analysis. Genes 11:786 DOI: 10.3390/genes 11070786 .

Ruiz-Herrera A, Nergadze SG, Santagostino M, Giulotto E. 2008. Telomeric repeats far from the ends: mechanisms of origin and role in evolution. Cytogentics Genome Research 122:219-228 DOI:10.1159/000167807.

Schober H, Ferreira H, Kalck V, Gehlen LR, Gasser SM. 2009. Yeast telomerase and the SUN domain protein Mps3 anchor telomeres and repress sub-telomeric recombination. Genes and Development 23:928-938 DOI: 10.1101/gad.1787509. 
324 Seluanov A, Chen Z, Hine C, Sasahara TH, Ribeiro AA, Catania KC, Presgraves DC,

325 Gorbunova V. 2007. Telomerase activity coevolves with body mass not lifespan. Aging Cell 326 6:45-52 DOI: 10.1111/j.1474-9726.2006.00262.x.

327 Tian X, Seluanov A, Gorbunova V. 2017. Molecular mechanisms determining lifespan in short328 and long-lived species. Trends in Endocrinology and Metabolism 28:722-734

Tian X, Doerig K, Park R, Can Ran Qin A, Hwang C, Neary A, Gilbert M, Seluanov A,

Gorbunova V. 2018. Evolution of telomere maintenance and tumour suppressor mechanisms across mammals. Philosophical Transactions of the Royal Society B: Biological Sciences 373:20160443 DOI: 10.1111/j.1474-9726.2006.00262.x

Van Daele PAAG, Dammann P, Meier JL, Kawalika M, Van De Woestijne C, Burda H. 2004.

Chromosomal diversity in mole-rats of the genus Cryptomys (Rodentia: Bathyergidae) from the $10.1017 / \mathrm{S} 0952836904005825$.

Van Lieshout SHJ, Bretman A, Newman C, Buesching CD, Macdonald DW, Dugdale HL. 2019. Individual variation in early-life telomere length and survival in a wild mammal. Molecular Ecology 28: 4152-4165 DOI: 10.1111/mec.15212.

Vleck CM, Haussmann MF, Vleck D. 2003. The natural history of telomeres: tools for ageing animals and exploring the ageing process. Experimental Gerontology 38:791-795 DOI: 10.1016/S0531-5565(03)00110-4.

Watson J. 1972. Origin of Concatemeric T7 DNA. Nature: New Biology 239:197-201 DOI: 10.1038/newbio239197a0. 
345 Yang F, Fu B, O’Brien PCM, Nie W, Ryder OA, Ferguson-Smith MA. 2004. Refined genome-

346 wide comparative map of the domestic horse, donkey and human based on cross-species

347 chromosome painting: insight into the occasional fertility of mules. Chromosome Research 1:65-

34876 DOI: 10.1023/B:CHRO.0000009298.02689.8a.

349 Weigl R. 2005. Longevity of Mammals in Captivity; from the Living Collections of the World.

350 Kleine Senckenberg-Reihe 48: Stuttgart

351 Zakian VA. 2012. Telomeres: The beginnings and ends of eukaryotic chromosomes.

352 Experimental Cell Research 12:1456-1460 DOI: 10.1016/j.yexcr.2012.02.015.

353 Zijlmans JM, Martens UM, Poon SSS, Raap AK, Tanke HJ, Ward RK, Lansdorp PM. 1997.

354 Telomeres in the mouse have large inter-chromosomal variations in the number of T2AG3 repeats.

355 Proceedings of the National Academy of Sciences 94:7423-7428 DOI: 10.1073/pnas.94.14.7423.

356 


\section{Figure 1}

Metaphase spreads of DMR, MMR and NMR chromosomes.

DNA is stained in blue (DAPI) and telomeres in red (Cy3 PNA probe). A. Metaphase spread of

DMR chromosomes. B.Partial metaphase spread of DMR chromosomes showing diffuse interstitial telomeric sequences (ITSs, arrowed, with middle arrow showing a very weak signal seen in some cells; see text). Note that some chromosomes are partially overlapping. C. Partial metaphase spread of MMR chromosomes. Note how some chromatids have double signals at the telomere, presumably representing differential condensation of the telomeric array (circles and expanded inset for one chromosome pair). A full image of this spread is given in Figure S1. D. Partial metaphase spread of NMR chromosomes. White arrow indicates a faint region of increased fluorescence that may be a potential region of ITSs. White scale bar $=10 \mu \mathrm{m}$; all images at the same magnification. 


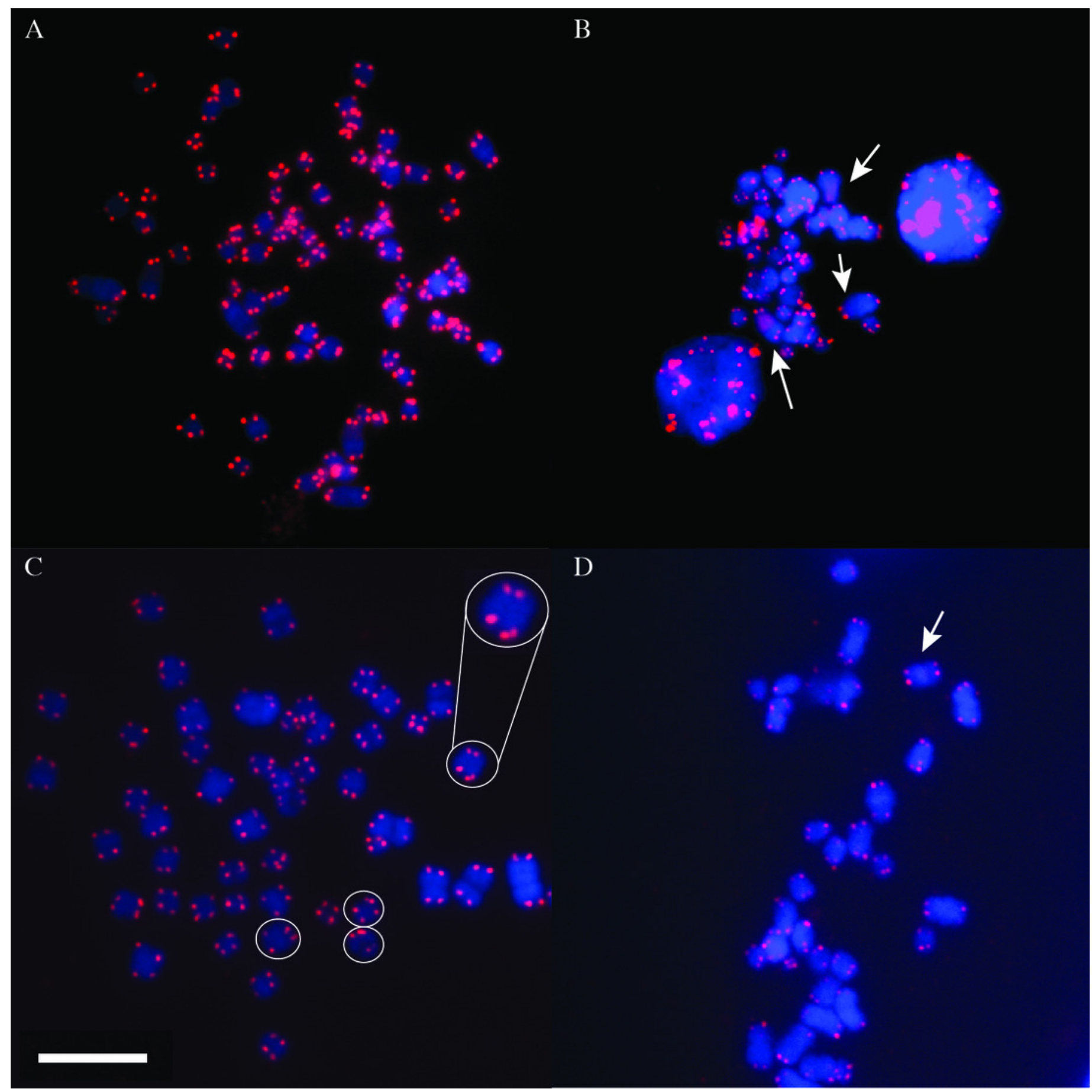


Figure 2

Boxplots depicting medians and interquartile ranges for telomere size (expressed as volume of telomere fluorescence) variation with age class.

A. DMR ( $n=6$ animals in each age class); B. MMR $(n=8,14$ and 6 individuals for young, middle-aged and old age classes respectively); C. NMR ( $n=3,2$ and 2 individuals respectively). 

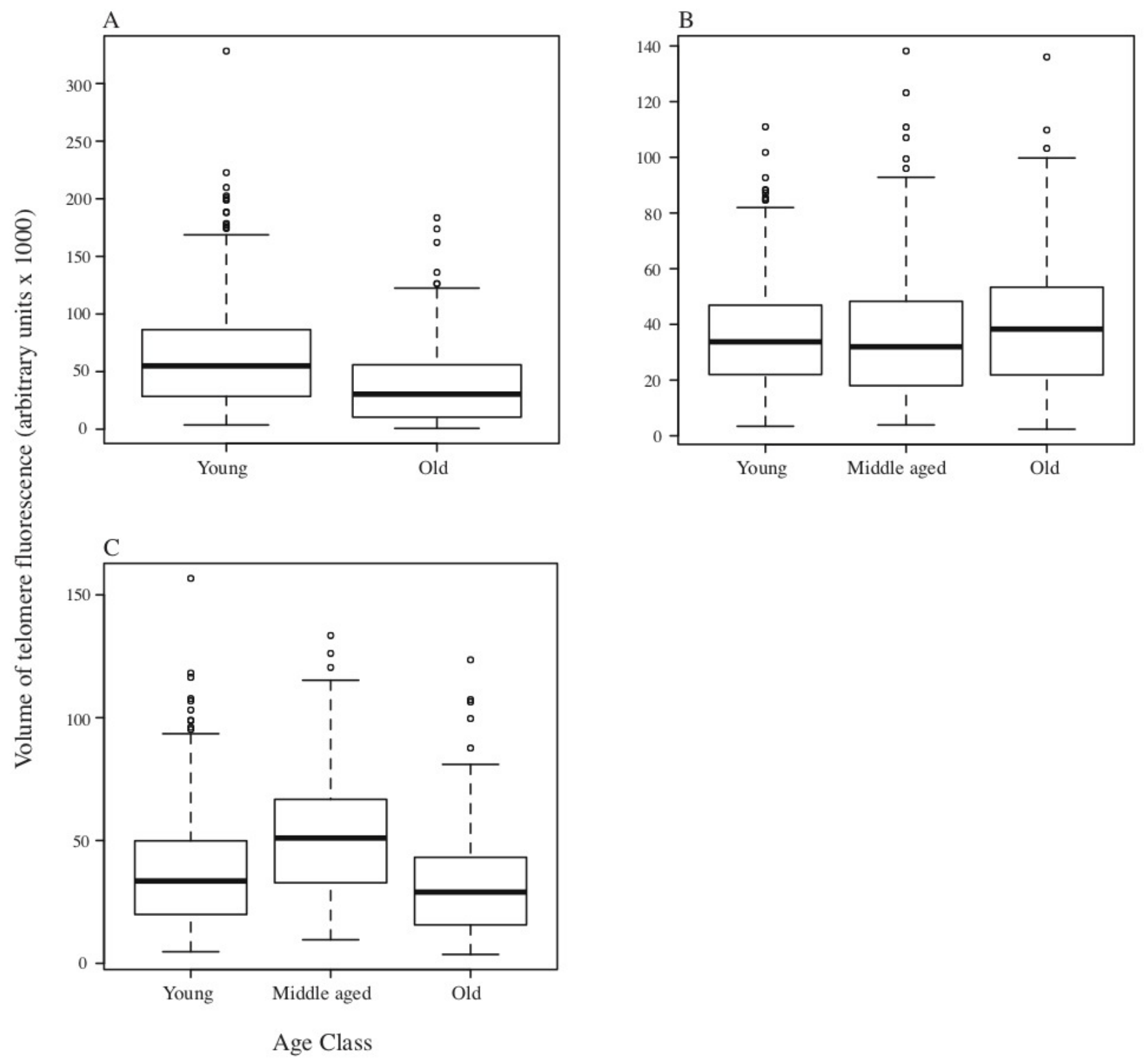